\title{
Laparoscopic Resection of a Retroperitoneal Liposarcoma: A Case Report and Review of the Literature
}

\author{
Ryohei Nomura, Hiromi Tokumura, Naoki Matsumura \\ Department of Surgery, Tohoku Rosai Hospital, Sendai, Japan
}

\begin{abstract}
Here, we describe a case of a retroperitoneal liposarcoma successfully managed by laparoscopic surgery. A 72-year-old man underwent abnormal hypertension screening using computed tomography (CT), which revealed a low-density mass measuring $7 \mathrm{~cm}$ in diameter in the retroperitoneal space. We diagnosed the mass as a liposarcoma before the operation. The mass was resected laparoscopically. No perioperative complications were encountered, and the patient was discharged on the fourth postoperative day. The pathologic diagnosis was well-differentiated liposarcoma, indicating complete surgical resection. Thus, we conclude that a laparoscopic approach for the patients with retroperitoneal tumors, including liposarcomas, is safe, feasible, and minimally invasive, even if there is a possibility of the tumor being malignant.
\end{abstract}

Key words: Retroperitoneal tumor - Liposarcoma - Laparoscopic surgery

L iposarcomas are rare, but are also the second most frequent retroperitoneal tumors, exceeded only by fibrous and fibrocystic malignancies. ${ }^{1}$ The World Health Organization (WHO) Committee for Classification of Soft Tissue Tumors in 2002 categorized soft-tissue liposarcoma into 5 types including well differentiated, dedifferentiated, myxoid, pleomorphic, and mixed. ${ }^{1,2}$ They often progress asymptomatically, and are detected incidentally. Surgical resection is the best single treatment for permanent cure of liposarcoma. ${ }^{3}$ Among the surgical proce- dures for retroperitoneal liposarcoma, open laparotomy has been widely accepted; however, only a few cases of laparoscopic approach have been reported. ${ }^{4-6}$ We report here a case of laparoscopic resection of a liposarcoma located in the retroperitoneum, and we review the literature.

\section{Case Report}

A 72-year-old man regularly visited the cardiology department of our institute because of chronic 


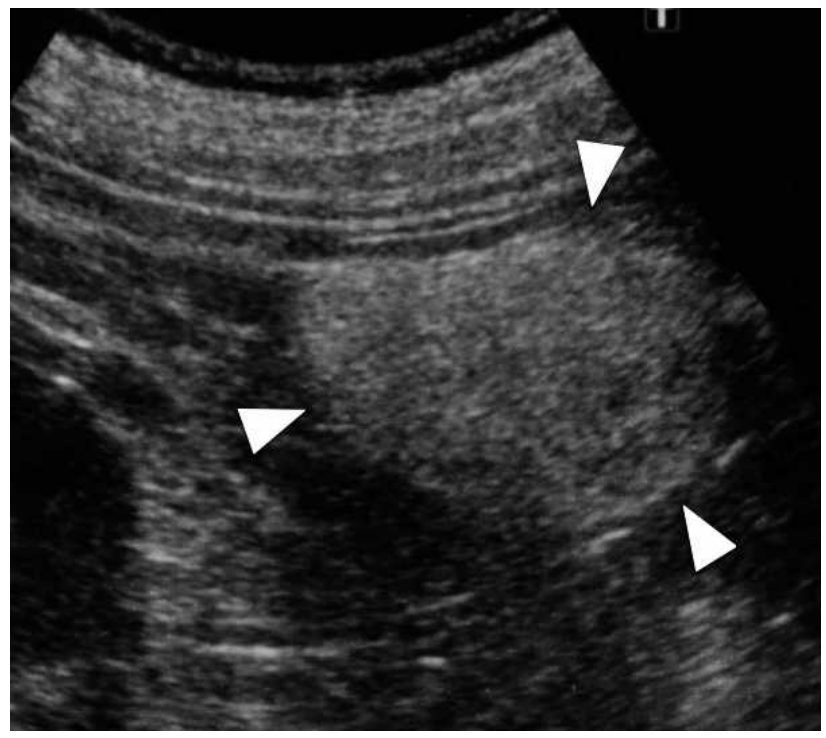

Fig. 1 The abdominal ultrasonography demonstrated hyperechoic mass (white arrow heads) measured $6.5 \mathrm{~cm}$ in longest diameter, which could be easily deformed by compressing.

hypertension and dyslipidemia. In May 2010, his primary doctor had prescribed screening computed tomography (CT) for abnormal hypertension. CT revealed a low-density mass measuring $7 \mathrm{~cm}$ in diameter in the retroperitoneal space incidentally. The patient was referred to our department for further treatment. The patient was asymptomatic; no palpable mass or abdominal discomfort was observed on physical examinations. Laboratory test results were within normal ranges, including the adrenal hormone levels. On image assessment, we re-evaluated the mass using abdominal ultrasonography and $\mathrm{CT}$ to obtain a pre-operative diagnosis. Abdominal ultrasonography revealed a hyperechoic mass measuring $6.5 \mathrm{~cm}$ in longest diameter (Fig. 1). The mass could be easily deformed by compressing, suggesting a soft tissue composition. In contrastenhanced CT, the mass was located in the retroperitoneal space, in the dorsum of the descending colon. The mass was slightly enhanced only in the upper level and was composed largely of lipomatous mass and nonlipomatous components with a septum. These findings led us to a pre-operative diagnosis of a well-differentiated liposarcoma (Fig. 2) occurring in the retroperitoneum. We thought it satisfactory to resect the mass laparoscopically, with adequate safety margins in case the mass was malignant. Under general anesthesia, the patient was placed in the lithotomy position. The first 12-

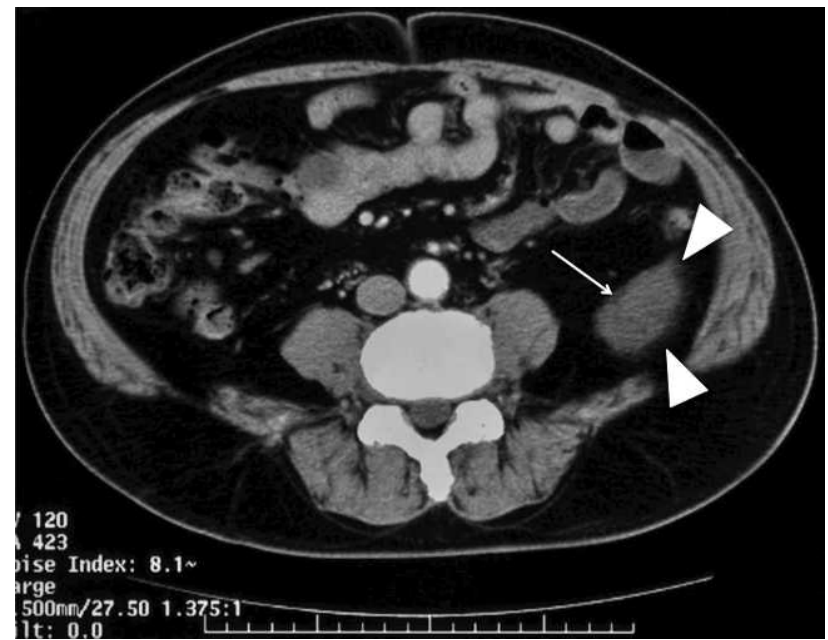

Fig. 2 The mass (white arrow heads) was composed of largely lipomatous mass and nonlipomatous components with a dividing septal structure (white arrow). The mass was diagnosed as welldifferentiated liposarcoma pre-operatively.

$\mathrm{mm}$ port was introduced above the umbilicus, by using the open laparotomy method. After creating a pneumoperitoneum by carbon dioxide insufflation, we inserted three 5-mm ports at the left lower and bilateral upper quadrant and a second $12-\mathrm{mm}$ port at the right lower quadrant (Fig. 3). We then mobilized the descending colon from the retroperitoneum to find a well-capsulated protruding mass. The mass was immediately exposed and widely dissected using laparoscopic coagulating shears (HARMONIC ACE, Ethicon Endo-Surgery, Cincinnati, Ohio) ensuring adequate safety margins (Fig. 4). The resected specimen was removed via a $4-\mathrm{cm}$ extended umbilical incision by using a laparoscopic removal bag (E:Z PURSE, Hakko, Nagano, Japan). We did not place drain tubes for confirming the absence of bleeding. All small wounds were closed subcutaneously. The operation time was $120 \mathrm{~min}$ and the estimated blood loss was minimal. No perioperative complications were encountered. The patient was put on a normal diet on the next day of the operation, and was discharged on the fourth postoperative day. The tumor was diagnosed as a well-differentiated liposarcoma histopathologically. Postoperative diagnosis by TNM classification ${ }^{6}$ was T2b, N0, M0, stage 1B.

No abdominal symptoms were observed and postoperative radiologic workup showed no evidence of recurrence during a 24-month follow-up period. 


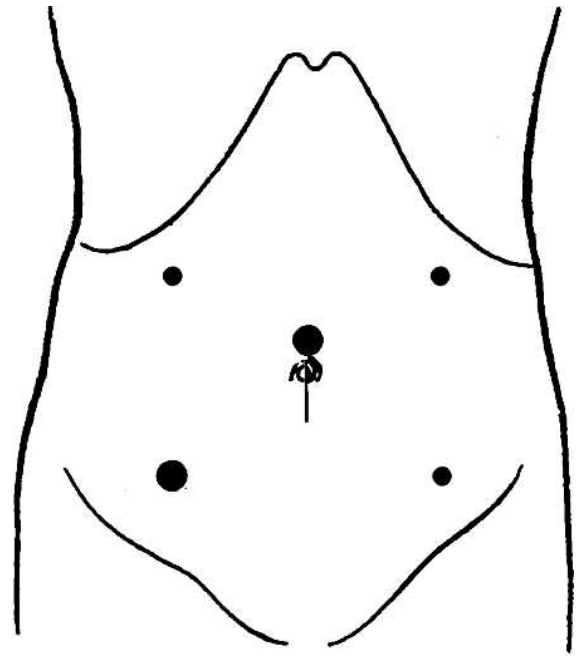

Fig. 3 Placement of ports: umbilicus (12-mm), right lower quadrant (12-mm), the left lower and bilateral upper quadrant (5$\mathrm{mm})$.

\section{Discussion}

Liposarcoma, the second most common malignant soft tumor, occurs mainly in the retroperitoneum, hip, and thighs. ${ }^{1}$ In retroperitoneal malignant soft tumors, it remains the most common. ${ }^{1,2}$ Liposarcomas often grow to a considerable size but are usually asymptomatic. Surgical management is the only permanent cure because complete resection with proper margins is strongly associated with recurrence and survival. ${ }^{3}$

With regard to surgical management, few reports of laparoscopic resection for retroperitoneal liposarcoma are available in the English literature. ${ }^{4-6}$ We have summarized the sporadic cases of laparoscopic approach for retroperitoneal liposarcoma in Table 1. Thus far, there were 3 women and 1 man (present case) with a mean age of 65.7 years (range, 53-77 years), have been reported. The range of maximal diameter and weight of the tumors was from 6.3-10 $\mathrm{cm}$ (mean, $8.8 \mathrm{~cm}$ ) and 20-380 g (mean, $200 \mathrm{~g}$ ), respectively. The tumors were located adjacent to the kidney in 2 cases, behind the cecum in 1 case, and behind the descending colon in the present one. The operation time and hospital stay was 120-150 min (mean operation time, $135 \mathrm{~min}$ ) and 4 days, respectively, including our case. With regard to the operative procedures, hand-assisted laparoscopic surgery was performed in one case, and laparoscopic management without hand-assist surgery was performed in the other 3 cases.

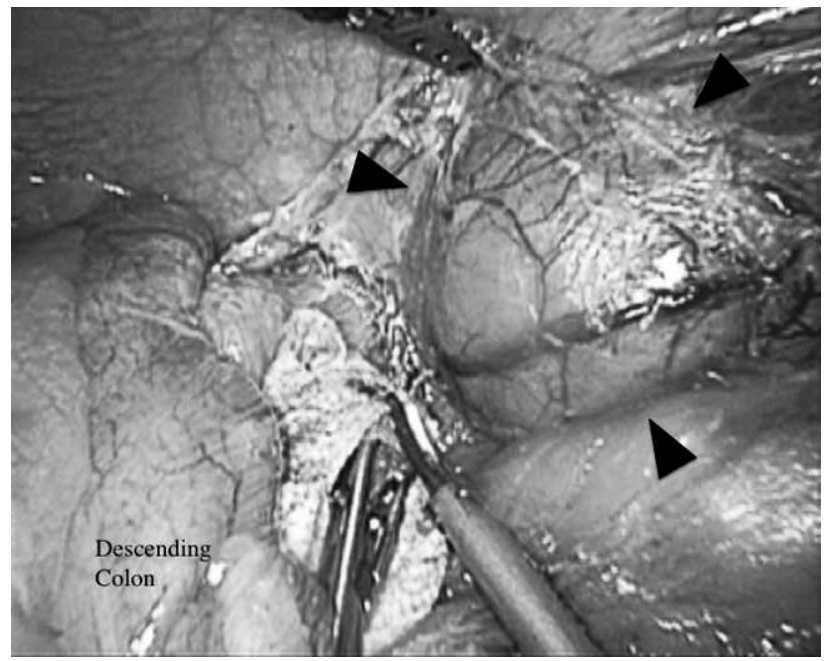

Fig. 4 A well-capsulated protruding mass (black arrow heads) was immediately exposed and widely dissected by laparoscopic coagulating shears, ensuring adequate safety margins.

For our patient, we chose a laparoscopic procedure for resecting the mass, because laparoscopic management of retroperitoneal mass has the advantages of less postoperative pain, rapid recovery, and better cosmesis. A laparoscopic approach would provide a better intra-operative view compared to the traditional open procedure. Moreover, we planned this approach with the intention of achieving complete removal of the mass and avoiding recurrence in the case of malignancy. Prior to cosmesis, maintaining the oncologic principles is the most important factor in choosing the operative method. From this point of view, we thought this maneuver was sufficient for removal of the mass and we could identify the encapsulated mass intra-operatively, since pre-operative ultrasonography or CT showed the mass to be ventrally protruding without any signs of invasion. These findings convinced us to identify the mass and ensure adequate safety margins of the mass. As expected, intra-operative findings showed an obvious demarcation line between normal retroperitoneal tissue and the mass that was protruding at the layer of the retroperitoneal subfascia. Thus, knowledge of the intraoperative appearance of the demarcation line enabled us to resect the mass completely. As laparoscopic maneuvers have the disadvantage of loss of tactile sensation compared with an open procedure, tumors must be visually apparent, especially in malignant tumors like liposarcoma. 
Table 1 Laparoscopic treatment of retroperitoneal liposarcoma

\begin{tabular}{|c|c|c|c|c|c|c|c|c|c|}
\hline Author (year) & $\begin{array}{l}\text { Age } \\
(\mathrm{yr})\end{array}$ & Sex & Location & $\begin{array}{l}\text { Size } \\
(\mathrm{cm})\end{array}$ & $\begin{array}{l}\text { Weight } \\
\text { (g) }\end{array}$ & $\begin{array}{l}\text { Operation } \\
\text { time (min) }\end{array}$ & $\begin{array}{l}\text { Hospital } \\
\text { stay (days) }\end{array}$ & Complication & $\begin{array}{r}\text { Pathologic } \\
\text { diagnosis }\end{array}$ \\
\hline Horiguchi (1998) & 53 & W & $\begin{array}{l}\text { Behind the cecum } \\
\text { Below the right } \\
\text { kidney }\end{array}$ & $10 * 10 * 7.5$ & 380 & NS & NS & $\begin{array}{l}\text { Port site } \\
\text { recurrence }\end{array}$ & myxoid \\
\hline Ball (2005) & 77 & W & $\begin{array}{l}\text { Adjacent to the left } \\
\text { kidney }\end{array}$ & $9 * 5 * 4.5$ & NS & NS & NS & none & well \\
\hline Dalpiaz (2007) & 61 & W & $\begin{array}{l}\text { Adjacent to the } \\
\text { right kidney }\end{array}$ & 10 & NS & 150 & 4 & none & well \\
\hline Present case & 72 & M & $\begin{array}{l}\text { Behind the } \\
\text { descending colon }\end{array}$ & $6.3^{*} 4.8^{*} 3.8$ & 20 & 120 & 4 & none & well \\
\hline
\end{tabular}

$\mathrm{W}=$ woman, $\mathrm{M}=$ man, NS = not stated, well = well-differentiated liposarcoma, myxoid = myxoid liposarcoma.

Since liposarcoma is known to be malignant, recurrence after resection is always a concern. ${ }^{8}$ In our case, we predicted that in case of a liposarcoma, subtype of the tumor would be well differentiated. This was based on the radiologic characteristics of the mass: lipomatous mass with nonlipomatous components in the thick septum. Murphy et al ${ }^{1}$ reported that well-differentiated liposarcomas frequently demonstrated a diagnostic appearance of a largely lipomatous mass and a nonlipomatous component in the thick septa or focal nodules. The diversity of the subtypes of liposarcomas is reflected in their clinical biologic behavior, which ranges from nonmetastasizing to high-grade neoplasms with extensive metastatic potential. ${ }^{3}$ Therefore, while considering laparoscopic management as treatment, pre-operative radiologic assessment is so essential. Laparoscopic surgery should be aggressively indicated in cases pre-operatively diagnosed as lowgrade liposarcoma; however, the surgery should be meticulously indicated in cases of high-grade neoplasms, like pleomorphic liposarcoma. Taken together, well-differentiated, small, and well-capsulated retroperitoneal liposarcoma is the best candidate for a laparoscopic approach.

We conclude that a laparoscopic approach for retroperitoneal liposarcoma is technically feasible and safe, and should be considered even if the tumors have a possibility of malignancy. However, the indication should be carefully reviewed by taking into consideration the size, location, and subtypes of the tumor.

\section{Acknowledgments}

The author wishes to acknowledge the kind assistance of Makoto Furihata, Department of Surgery, the Mutual Aid Association for Teachers and Officials Sanraku Hospital, in editing the manuscript. None of the authors have conflicts of interest to disclose, and have no sponsorship or funding arrangements relating to our research.

\section{References}

1. Murphy MD, Arcara LK, Fanburg-Smith J. From the archives of the AFIP: imaging of musculoskeletal liposarcoma with radiologic - pathologic correlation. Radiographics 2005;25(5): 1371-1395

2. Christopher D, Unni K, Mertens F, eds. WHO classification of the tumors. Pathology and genetics of tumors of soft tissue and bone. Chapter 1, Adipocytic Tumors. Lyon, France: IARC, 2002: $19-46$

3. Singer S, Antonescu CR, Riedel E, Brennan MF. Histologic subtype and margin of resection predict pattern of recurrence and survival for retroperitoneal liposarcoma. Ann Surg 2003; 238(3):358-371

4. Horiguchi A, Saito S, Baba S, Murai M, Mukai M. Port site recurrence after laparoscopic resection of retroperitoneal liposarcoma. J Urol 1998;159(4):1296-1297

5. Ball AJ, Siddiq FM, Garcia M, Ganjei-Azar P, Leveillee RJ. Hand-assisted laparoscopic removal of retroperitoneal liposarcoma. Urology 2005;65(6):1226

6. Dalpiaz O, Gidaro S, Lipsky K, Schips L. Laparoscopic removal of 10-cm retroperitoneal liposarcoma. J Endourol 2007;21(1):8384

7. Sobin LH, Gospodarowicz MK, Wittekind Ch, eds. TNM classification of malignant tumors. In: O'Sullivan B, ed. Soft Tissue Tumor. 7th ed. New York: Wiley \& Blackwell, 2010:147151

8. Bautista N, Su W, O'Connell TX. Retroperitoneal soft-tissue sarcomas: prognosis and treatment of primary and recurrent disease. Am Surg 2000;66(9):832-836 